\title{
Evaluation of 1D Selection Techniques for Mobile Visualizations
}

\author{
MICHAIL SCHWAB, ADITEYA PANDEY, and MICHELLE A. BORKIN
}

In spite of growing demand for mobile data visualization, few design guidelines exist to address its many challenges including small screens and low touch interaction precision. Both of these challenges can restrict the number of data points a user can reliably select and view in more detail, which is a core requirement for interactive data visualization. In this study, we present a comparison of the conventional tap technique for selection with three variations including visual feedback to understand which interaction technique allows for optimal selection accuracy. Based on the results of the user study, we provide actionable solutions to improve interaction design for mobile visualizations. We find that visual feedback, such as selection with a handle, improves selection accuracy three- to fourfold compared to tap selection. With a $75 \%$ accuracy, users could select a target item among 176 items total using the handle, but only from 60 items using tap. On the other hand, techniques with visual feedback took about twice as long per selection when compared to tap. We conclude designers should use selection techniques with visual feedback when the data density is high and improved selection precision is required for a visualization.

CCS Concepts: • Human-centered computing $\rightarrow$ Empirical studies in HCI.

Additional Key Words and Phrases: Mobile, Interaction Design, Human-Subjects Quantitative Studies

ACM Reference Format:

Michail Schwab, Aditeya Pandey, and Michelle A. Borkin. 2021. Evaluation of 1D Selection Techniques for Mobile Visualizations. In Schwab '21: ACM CHI, fune 03-05, 2021, Woodstock, NY. ACM, New York, NY, USA, 10 pages. https://doi.org/10.1145/XXXXXXX.XXXXXXX

\section{INTRODUCTION}
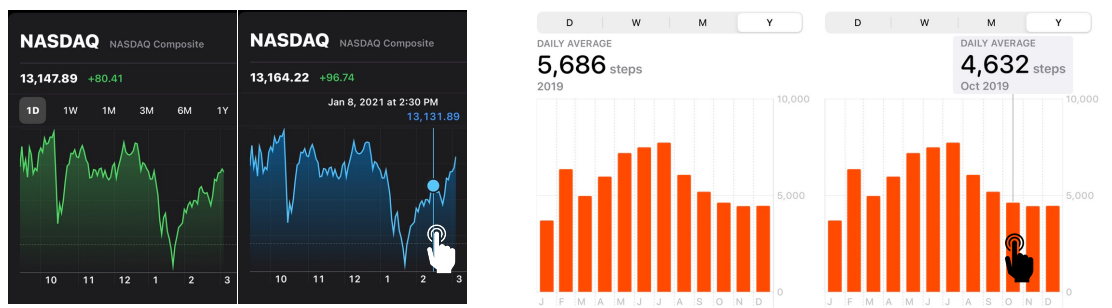

Fig. 1. Left: A timeline visualization representing the variation in the NASDAQ index price. Interactivity reveals additional information such as the NASDAQ index's price at a particular time of the day. Right: A bar chart visualization that shows the average number of steps taken by a user in a year. Tapping over a bar shows the average number of steps taken in a month.

Data visualizations have become commonplace on mobile devices: calendars present events for the day, the news display context for articles, stock charts help people make financial decisions (Figure 1: Left), and fitness visualizations are used to improve health, such as in the number of steps taken in a day (Figure 1: Right). The design of visualizations for mobile devices presents many challenges to visualization creators. For example, a common problem on mobile visualizations is the small display size of mobile devices which precludes designers from showing everything at once in a single view.

Permission to make digital or hard copies of all or part of this work for personal or classroom use is granted without fee provided that copies are not made or distributed for profit or commercial advantage and that copies bear this notice and the full citation on the first page. Copyrights for components of this work owned by others than ACM must be honored. Abstracting with credit is permitted. To copy otherwise, or republish, to post on servers or to redistribute to lists, requires prior specific permission and/or a fee. Request permissions from permissions@acm.org.

(C) 2021 Association for Computing Machinery.

Manuscript submitted to ACM 


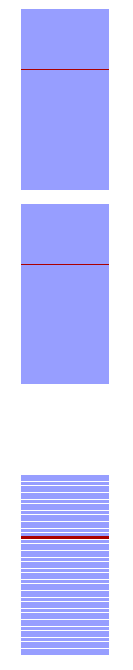

Visual Feedback Makes Selection Slower But More Accurate

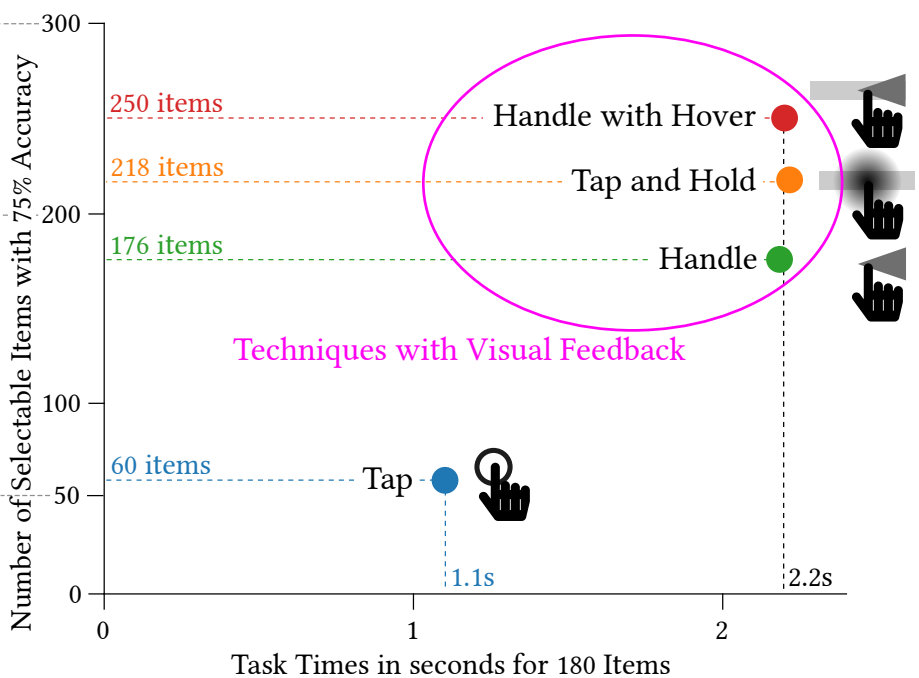

Fig. 2. On mobile touch screens, selection techniques which allow users to refine their selection before committing to a selection consistently perform 3-4 times more accurately than tap, and twice as slowly. Such techniques include Handle, where a handle is dragged onto a target item, Tap and Hold, where item-level hover feedback is provided while holding down the finger, and Handle with Hover, where the draggable handle additionally provides item-level hover feedback.

Therefore, it is common practice to provide more information through interactive techniques such as tapping or pressing and holding a data point (Figure 1). As a result, an effective interaction technique can be a critical factor in the design of a mobile visualization and can separate a visualization that frustrates the user from a visualization that supports a user's goals. Therefore, in this paper, we ask: How can we design mobile data visualization interactions to be more effective?

Unfortunately, insufficient work has been done to understand and evaluate mobile interaction for visualizations that require 1D selection of items such as a timeline or a bar chart. On mobile, the limited screen space and the low precision of tap interactions make it hard for users to select small items, constraining the number of selectable items on a visualization. To select a target item among thousands, visualization creators can utilize multiscale techniques, such as pan and zoom, as well as more accurate selection techniques. However, it is unclear what strategies visualization creators should choose: Should drag-based selection be preferred? How many items can visualization creators display without needing a multiscale environment? To provide visualization creators with useful guidance, studies must be run which are applicable to the Web-a highly uncontrolled environment accessed through a multitude of devices.

We contribute a broadly applicable novel study on simple single-touch 1D selection techniques to understand which interactions should be used by visualization creators to enable users to interact with many data points. This study is the first evaluation to report the effect of single-scale selection techniques on mobile navigation. We compare the conventional tap selection with three variations of the main alternative selection technique, drag-based selections. We find that all three techniques which provide visual feedback allow users to select items three to four times more accurately than with tap, demonstrating that these techniques enable users to interact on small screens with more data on visualizations. On the other hand, using these alternative techniques took longer for the users to execute, about 2.2 seconds on average compared to 1.1 seconds with tap for comparable tasks (Figure 2). We discuss this accuracy versus efficiency trade-off and its impact on the design of mobile visualizations. Our study data and analysis routines are provided in the Supplemental Material. 


\section{RELATED WORK}

\subsection{Mobile Data Visualization and Challenges}

Researchers have studied mobile visualizations in many contexts. Recent work has identified and evaluated appropriate visual encodings to display data on small screen devices $[8,11,12,40]$. Another research area in mobile visualizations is the use of visualizations on small screen devices to support navigation or analysis of data on large screen devices [7, 24, 37]. Some studies investigated interaction methods for mobile visualizations. For example, Balaa et al. [4] proposed an interaction technique called "DicthoZoom" to select objects in a dense 3D Virtual Environment and compared it with other interaction modalities on smartphones. In another work, Krone et al. [27] study interactions on mobile devices for scientific visualizations. However, the current work in mobile visualization interaction design lacks design guidelines for more broadly useful visualizations like timelines and bar charts, which depend on the 1D selection and interaction methods.

Mobile data visualization comes with challenges that make effective navigation harder to achieve than on desktop computers. While some related work provide a good overview of the problems in mobile visualization [16], there are few with explicit solutions to these challenges. At VIS, four papers about visualization on mobile devices have been presented in total since 2013 [11, 12, 28, 40]. The MobileVis workshop held at CHI 2018 [1] discussed the various types of challenges, yet few solutions were presented and established the need for more design guidelines for mobile visualization.

Two significant design challenges for mobile data visualization are screen size and pointing precision, in part due to occlusion $[15,16,21,29,41]$. The combination of the challenge of fingers occluding parts of the relatively small interfaces, and the low accuracy of touch input, is referred to as the "fat finger problem" [36]. One area of related work reduces the need for precise interaction through effective summarization techniques and proposes more use of the "overview first, details later" mantra on mobile [14]. One common approach to execute this strategy is to use multi-scale techniques, such as pan and zoom, where users can drill down on relevant areas of the visualization [6, 9, 34, 35, 39]. However, it is unclear when it is necessary and appropriate to use multi-scale techniques, and when single-scale selection methods are preferable, as not enough clarity exists on the efficiency of single-scale techniques.

For single-scale selection techniques, visualization creators can choose between tap-based techniques, drag-based techniques, and hybrids [34]. One area of research targets the interaction precision with device designs, such as touch interactions occurring on the back of a device rather than on the front screen, to prevent occlusion [5, 41]. For tap-based techniques, some related work identifies and reduces systematic errors in touch locations [23], and other work improves accuracy by using multiple taps for multi-scale [34]. For drag-based techniques, Potter et al. compared error rates between drag and tap when selecting a target item among a grid of $5 \times 10$ items, and found the error on drag to be significantly lower [31]. Cockburn et al. compare tap with stylus and mouse [17], but also focus their research on selecting among relatively few items.

Unfortunately, most accuracies reported are only representative for the specific number of items displayed in the study. Existing studies only capture a snapshot of the range of accuracies that result from users interacting with a range of items. Therefore, little is known about how many items can be reliably distinguished with tap and drag-based techniques. This is crucial knowledge for visualization creators, as they need to choose interaction techniques based on the amount of data their users are expected to navigate.

\subsection{Common Visualization Techniques with 1D Selection Tasks}

This section introduces two common visualization techniques that can use 1D-selection of data.

Timelines: Chronology in events has been communicated through timelines for centuries [30, 32]. Today, timeline visualizations have become commonplace on mobile devices, such as on calendars, in the news, and photo applications. 
Brehmer recently provided a summary of various timeline shapes, including linear, radial, grid, spiral, and arbitrary [10]. This helps us comprehend visual choices, but little is known about their consequences: which shape easiest to interact with? Some recent works compare timeline designs in terms of understandability or effectiveness [3,11, 18, 35, 38], and some investigate the effect of tooltips on interaction on desktop [2], yet performance in most of the design space is still unknown, especially on mobile.

Bar Charts: Bar charts are another common visualization technique that can utilize 1D item selection for navigation and exploration of the visualization. Bar charts are common on mobile devices for health and fitness apps and in data journalism [33]. Bar charts are conventionally drawn as a vertical columns a horizontal bars, but, similar to timelines, little information is available on how to interact with these charts on mobile.

Visualizations that require 1D selection of items for interaction and navigation are becoming increasingly popular on mobile devices. However, there are no existing studies to evaluate different methods of interaction that can be effective for such visualizations. Therefore, we present a study to evaluate four interaction techniques that can be used for 1D selection tasks (Section 3). Our study is the first evaluation to report the effect of single-scale selection techniques on mobile navigation, a critical component of visualizations with 1D selection tasks.

\subsection{Motor Control Theory}

Motor control theory is useful to describe the time needed for the physical pointing work in item selection tasks. Fitts' Law [20] models the time $T$ for a pointing task to navigate to a target of a specific size at a known distance using the techniquedependent parameters $a$ and $b$. The relationship between distance and target size is called the index of difficulty (ID).

$$
T=a+b \cdot \mathrm{ID}=a+b \cdot \log _{2}\left(\frac{\text { Distance }}{\text { TargetSize }}+1\right)
$$

To make our results transferable to other visualizations, we model performance using Fitts' law. For tap selection, we find that task times are mostly uncorrelated with task difficulty, shown in Figure 4 (right). For drag techniques, the mean task time increases linearly with the index of difficulty, as predicted by Fitts' law. This shows that even in our uncontrolled study setting, results generally agree with the theory. The agreement validates the approach and aligns with previous results [35].

\section{STUDY DESIGN AND PROCEDURE}

The goal of this mobile study is to evaluate the number of items from which users can still select a target item reliably using touch interaction on a mobile device, and how this varies by selection technique. Specifically, we set out to answer whether and how much the visual feedback of drag techniques can improve selection accuracy. We compare the ubiquitous tap technique with simple drag-based techniques, and vary the type of visual feedback given during drag to see which type of visual feedback improves accuracy, if any.

\subsection{Study Design}

Crowdsourced Study. We seek practical insights into which interactions perform best given the wide range hardware in use, as opposed to well-defined laboratory settings which may or may not be applicable in the real world (see [19]). Our study is run on Amazon's Mechanical Turk and does not control user nor input devices to conserve the real-world variability faced web application designers today. Smart phones were required to be used in portrait mode - simulating the common scenario of an initial Webpage load, where smart phones are in portrait mode about $90 \%$ of the time [25]. 

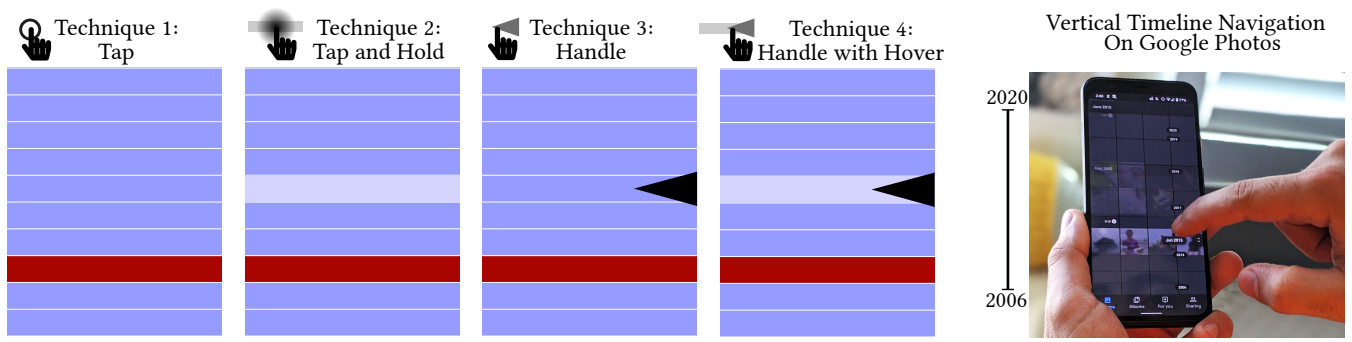

Fig. 3. Left: Each condition of the experiment requires the user to select a target item, shown in red; here, the user must select one item from ten. The experiment evaluates the precision of four different selection techniques. Technique 1 selects an item with a single tap. Technique 2 is based on tap and hold to provide item-level hover feedback during the selection, supporting corrections the selection. Technique 3 uses a handle that can be dragged up and down. Technique 4 is a combination of techniques 2 and 3 , as both a handle and item-level hover feedback are provided. Right: Google Photos is a practical example of a densely populated vertical timeline on mobile, displaying thousands of photos and many years across the height of a mobile phone. Google Photos uses handle-based drag selection, similar to Technique 3 .

Task and Visual Design. A summary of the four experiment conditions is shown in Figure 3. Using the typology by Brehmer et al. [13], the task is a target known, location known lookup task. We use a simple list design that is common for both timeline and bar charts to represent items for generalizability and to avoid distractions. This list design is an abstraction of a vertical timeline where users need to select items on the timeline with a certain accuracy - in effect, the list is a visualization of the hit boxes of items on a timeline. We chose this visualization design because it most directly and simply represents the underlying task of selecting a time frame of a specific length.

Interactions. We compare the ubiquitous Tap gesture (Figure 3) with three drag techniques that provide visual feedback (Figure 3, techniques 2-4). In addition to the overall effect of visual feedback, we set out to understand what kind of visual feedback is most helpful: Drag provides continuous position feedback which can be used to either directly display this position to the user, or to highlight currently hovered items. We compare a $\$$ Handle, which gives visual feedback over the current position, with item-level feedback $\$$ Hover. We use $\$$ Handle with Hover to learn if the two techniques are beneficial together. Below we explain in more detail the evaluated interaction techniques, and demonstrate the techniques in the Supplemental Video:

1. Tap : In the tap interaction, participants select an item on a touch interface with their finger. This technique is ubiquitous, direct, simple, and fast. However, no visual feedback is provided.

2. \$ Hover : This interaction is a tap interaction with item-level visual feedback about the currently selected item. Users long-press an item to select. We set a minimum hold time of $300 \mathrm{~ms}$.

3. Handle : This interaction is inspired by a desktop mouse and comparable with the Offset Cursor [31], but takes advantage of the 1D nature of visualizations by using a handle. Selection is done by tapping, holding, dragging, and releasing the handle. This technique has an additional time cost because the handle has to be selected before navigating to the target. In terms of Fitts' law, an additional distance has to be covered. There is visual feedback for the current pointer position, but there is no item-level feedback.

4. Handle with Hover : This interaction is a combination of $\$$ Handle and Hover . The selection is shown via the handle and through item-level visual feedback.

The study has four factors and was run within-subjects. The conditions appeared in random order to counter learning effects. To measure the selection precision,we choose a vertical layout with items covering the full width (see Figure 3 ). Participants are asked to select a randomly assigned target. We increase the number of items on the screen after a correct 
answer and decrease the number of items after an incorrect answer, in effect making individual items larger and smaller, respectively. The amount by which items are increased and decreased is determined by a three-down, one-up staircase, as suggested by García-Pérez et al. [22] and applied in data visualization by Kale et al. [26]. On balance, this 3-down, 1-up ladder stabilizes around a $75 \%$ accuracy (since $\frac{3}{4}=0.75$ ). For our analysis, we compare the maximum number of items that can be resolved at this accuracy across selection techniques. The displayed number of items corresponds to a task difficulty, measured in the index of difficulty as modeled by Fitts' law (see Section 2).

Hypotheses. Our main hypothesis was that $\&$ Tap performs less accurately than all three other techniques because it does not provide visual feedback and does not allow users to fine-tune their selection. Our secondary hypothesis was that $\$$ Handle with Hover, with its visual feedback about both position as well as item-level hover feedback, would perform slightly better than the other techniques.

Task Design. For each condition, participants had to complete four test phase tasks correctly before we collected their data. Participants started at a fixed difficulty of 10 items, and gradually increased the number of items by making correct selections until the task became too difficult. Through pilot testing, we found that participants reached their maximum number of displayed items around task number 32 . We set the number of tasks per condition to 40 .

Participants and Data Recorded. We recruited 50 participants. This number was determined after a pilot test with 8 participants. Of the 50 participants, 16 were female, 33 male, and one participant did not disclose their gender. One participant was 18-24 years old, 29 participants were 25-34, 17 participants were 35-44, 2 participants were 55 years old and above, and one participant did not disclose their age. Participants were required to be located in the United States, have a HIT Approval Rate greater than or equal to $98 \%$, and have more than 500 approved HITs. Each participant was told of the duration, pay, procedure, and goal of the study, informed of their rights, and asked for their informed consent through our institution-approved IRB protocol. The median time to complete the study was 9 minutes 40 seconds. Each participant was compensated $\$ 3.00$.

All participants were asked to perform the task on their smart phone. We recorded quantitative study data, device information, optional demographic information (age, gender), and optional participant feedback. The study data includes correctness of each task and time taken to complete each selection task.

Outlier Removal. It is well-established that studies run on Amazon's Mechanical Turk include participants that pay less attention than participants in laboratory settings [19]. However, due to the simplicity and short time of each selection task (typically less than 5 seconds), we expect participants' accuracy to be less affected by a lack of attention than their task times. We checked the data for participants that do not appear to try to select the target items accurately, but found no such case, perhaps due to our inclusion criteria. Therefore, for correctness and accuracy calculations, no outliers are removed. To address participants taking breaks during a task, we remove task times that are two times the standard error larger than mean task times. This removed $19 \%$ of task time data, a high amount typical for this uncontrolled setting.

\subsection{Analysis}

To compare the techniques, we obtain the difficulty of tasks at which participants could still perform reliably. For our analysis, we choose to evaluate data at the $75 \%$ accuracy mark because this is where the 3-up, 1-down ladder stabilizes. For different selection criteria, analyses for other accuracies can be performed via our open science data. To calculate the accuracy per difficulty, we count the number of correct tasks and divide them by the number of total tasks in a range of 2 bits of difficulty around the specified difficulty. This method aggregates selection tasks from participants that experienced tasks to be at different difficulties due to their varied device sizes and resolutions, which results in different task difficulties. From 

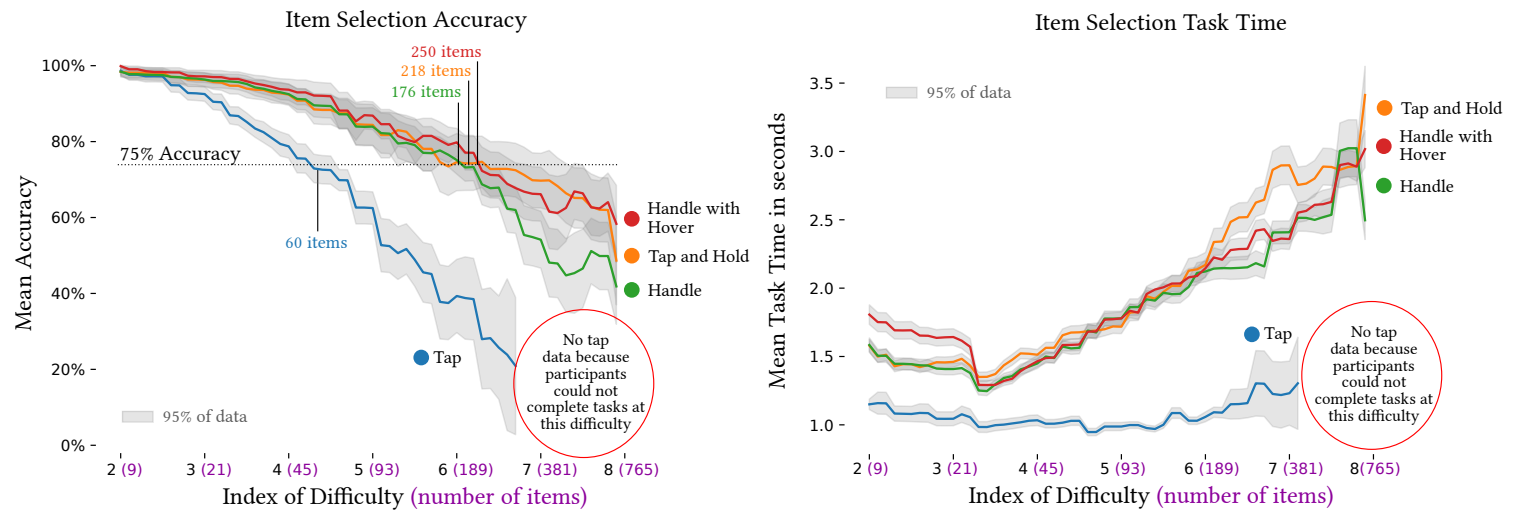

Fig. 4. Left: Technique accuracy comparison for varying task difficulties. Across all difficulties, participants had lower accuracy with Q Tap than with all drag-based techniques. For very difficult tasks, no data is available for tap because participants did not reach these difficulties because they made too many incorrect selections with tap. For tap, participants were able to select the target from 60 items at a $75 \%$ accuracy. For the 5 Handle , Hover, and Hande with Hover 176, 218, and 250 items at a $75 \%$ accuracy, respectively. Right: Technique task time comparison for varying task difficulties. Selection tasks are performed the fastest with tap across all difficulties, and task time does not increase much with task difficulty for tap, whereas participants take longer to complete more difficult tasks on the other techniques.

the aggregation, we obtain one index of difficulty per participant and technique, or four values per participant. Altogether, we measure 50 sets of difficulties for each technique. We perform a two-sided $t$-test for all 6 combinations of technique pairings. We set the family-wise error rate at $5 \%$. With a Bonferroni correction of 6 , the individual $\alpha$ is set to $0.05 / 6=0.008$.

\section{RESULTS}

On average, as shown in Figure 4 (left), the maximum index of difficulty at 75\% accuracy was 4.4 for $\$$ Tap , 5.9 for $\$$ Handle , 6.2 for $\$$ Hover, and 6.4 for $\$$ Handle with Hover. This corresponds to $60,176,218$, and 250 items at which participants could still resolve the target at a $75 \%$ accuracy for $\$$ Tap , $\$$ Handle , \$ Hover, and $\$$ Handle with Hover , respectively. Hence all 3 techniques with visual feedback could discriminate 3-4 times the number of items from the target in a single interaction compared to Tap. The three $p$-values comparing $\&$ Tap with the other techniques are all $<0.008$, indicating significant differences. Comparing $\$$ Hover with $\$$ Handle , \$ Hover with $\$$ Handle with Hover, and $\checkmark$ Handle with $\$$ Handle with Hover, we do not find significant differences, with $p$-values of $0.16,0.50$, and 0.03 , respectively (see subsection 3.2 for $\alpha$ values). The hypothesis that the visual feedback of techniques $2-4$ enables more accurate selection holds true. The secondary hypothesis that the handle with item-level hover outperforms the other technique is inconclusive, as this technique does have a higher average accuracy, but does not show a significant difference in this study.

To understand time trade-offs, we include statistics about time for task completion. These results are not significancetested since this study primarily evaluates accuracy. As shown in Figure 4 (right), Tap is the fastest technique across all difficulties, and task time does not increase with difficulty. However participants were unable to perform tasks harder than an index of difficulty of 6, corresponding to about 190 items, whereas with the other techniques participants were sometimes able to complete tasks up to an index of difficulty of 8, or 765 items. Drag-based techniques behave similarly and the time to completion increases with task difficulty. Even for very difficult tasks with over 700 items, participants performed the task within 3-4 seconds. 


\section{DISCUSSION}

Our results suggest a design trade-off for interactive selection tasks: that visual feedback can triple to quadruple selection accuracy at the cost of an additional 0.5 seconds for easy tasks and up to one additional second for moderately difficult tasks. While participants generally take more time for their selections as difficulty increases, task times for the tap selection is near-constant (Figure 4, right) because tap does not provide a way for users to take advantage of more time. After an index of difficulty of 3 bits, or 21 items, the accuracy of tap begins to drop in comparison to the other techniques.

Visual feedback about the current position and visual feedback about the currently hovered item appear to perform similarly. When combined, as with $\$$ Handle with Hover, the precision is slightly increased, but we find no significant difference. The $\$$ Hover, which only uses item-level hover for visual feedback, had higher task completion times. This may indicate that participants take more time validating their selection before releasing the interaction. Task times include a "dip" around an index of difficulty of 3.3. We believe this to be a learning effect, as this is the first point in the graph that does not include data from the first recorded task, where participants may have been familiarizing with the tasks and selection techniques. This is supported by the fact that the drop in task times is smaller for the more familiar tap selection technique.

This study was run in a 1D setup with a set of four particular interactions. Based on the similar results among the three drag-based techniques, we suspect that the results generalize to other $1 \mathrm{D}$ drag-based selections. For 2D visualizations, smaller handles are needed to avoid obscuring items. Solutions for the obstruction challenge need to be considered, such as using an offset between finger and handle (see Offset Cursor [31]).

This study also assumes a setup in which participants complete task after task with a clearly marked target. In realistic situations, participants would have to first focus and understand the interface and then find the target item before performing the selection task analyzed in this study. For this reason, visualization creators should be aware that the practical time difference between tasks using tap and the other techniques might be larger than our stated selection times.

Our results were obtained in a crowdsourced study. The variability of input devices and usage contexts across participants improves the study's external validity as it is closer to how a visualization with 1D Selection task would be accessed on the Web today. While we only have data for the four techniques we measured, the similarity across all techniques with visual feedback and their very consistent outperforming of tap leads us to believe that it is most important to include visual feedback in some form to allow much better selection accuracy than with tap selection. However, the increased time it takes to use handle-based interaction techniques suggests that combinations of these techniques could bring the advantage that for easy tasks, users can simply tap, whereas for difficult selections, they can refine using the handle. Such combinations should be evaluated in future research.

\section{CONCLUSION}

Mobile visualization is challenging and compromises have to be made to allow effective navigation given smaller screens and lower interaction accuracy. In our study on 1D visualizations, we have compared traditional tap selection with drag-based techniques that provide visual feedback to refine the interaction. We have provided evidence that these techniques perform similarly and all allow users to select a target item from 3-4 times more items than with tap. Drag techniques are slower than tap, but average total task times were still only about 3 seconds even for the hardest tasks that participants were unable to do with tap. Beyond our evaluation, we hope readers can learn from our recognition that the number of reliably selectable items is important to optimize on mobile data visualizations. All data and data analysis routines used are open source at osf. io/spmv9. Future research can build on this work for repeatable and reproducible user studies to improve navigating mobile visualizations. 


\section{REFERENCES}

[1] ACM 2018. Proc. Mobile Visualization Workshop. ACM, Montreal, Canada.

[2] Muhammad Adnan, Mike Just, and Lynne Baillie. 2016. Investigating Time Series Visualisations to Improve the User Experience. In Proceedings of the 2016 CHI Conference on Human Factors in Computing Systems (San Jose, California, USA) (CHI '16). Association for Computing Machinery, New York, NY, USA, 5444-5455. https://doi.org/10.1145/2858036.2858300

[3] Danielle Albers, Michael Correll, and Michael Gleicher. 2014. Task-Driven Evaluation of Aggregation in Time Series Visualization. In Proceedings of the SIGCHI Conference on Human Factors in Computing Systems (Toronto, Ontario, Canada) (CHI '14). Association for Computing Machinery, New York, NY, USA, 551-560. https://doi.org/10.1145/2556288.2557200

[4] Eliane Balaa, Mathieu Raynal, Florent Cabric, and Emmanuel Dubois. 2018. DichotoZoom: Selection Technique in a 3D Virtual Environment Displayed on Smartphone. In Proceedings of the 30th Conference on l'Interaction Homme-Machine (Brest, France) (IHM '18). Association for Computing Machinery, New York, NY, USA, 108-118. https://doi.org/10.1145/3286689.3286695

[5] Patrick Baudisch and Gerry Chu. 2009. Back-of-device Interaction Allows Creating Very Small Touch Devices. In Proceedings of the SIGCHI Conference on Human Factors in Computing Systems (Boston, MA, USA) (CHI '09). ACM, New York, NY, USA, 1923-1932. https://doi.org/10.1145/1518701.1518995

[6] Hrvoje Benko, Andrew D. Wilson, and Patrick Baudisch. 2006. Precise Selection Techniques for Multi-touch Screens. In Proceedings of the SIGCHI Conference on Human Factors in Computing Systems (Montr\&\#233;al, Qu\&\#233;bec, Canada) (CHI '06). ACM, New York, NY, USA, 1263-1272. https://doi.org/10.1145/1124772.1124963

[7] Lonni Besançon, Mickael Sereno, Lingyun Yu, Mehdi Ammi, and Tobias Isenberg. 2019. Hybrid Touch/Tangible Spatial 3D Data Selection. Computer Graphics Forum 38, 3 (2019), 553-567. https://doi.org/10.1111/cgf.13710 arXiv:https://onlinelibrary.wiley.com/doi/pdf/10.1111/cgf.13710

[8] T. Blascheck, L. Besançon, A. Bezerianos, B. Lee, and P. Isenberg. 2019. Glanceable Visualization: Studies of Data Comparison Performance on Smartwatches. IEEE Transactions on Visualization and Computer Graphics 25, 1 (Jan 2019), 630-640. https://doi.org/10.1109/TVCG.2018.2865142

[9] Sebastian Boring, David Ledo, Xiang "Anthony" Chen, Nicolai Marquardt, Anthony Tang, and Saul Greenberg. 2012. The Fat Thumb: Using the Thumb's Contact Size for Single-Handed Mobile Interaction. In Proceedings of the 14th International Conference on Human-Computer Interaction with Mobile Devices and Services (San Francisco, California, USA) (MobileHCI '12). Association for Computing Machinery, New York, NY, USA, 39-48. https://doi.org/10.1145/2371574.2371582

[10] Matthew Brehmer, Bongshin Lee, Benjamin Bach, Nathalie Henry Riche, and Tamara Munzner. 2017. Timelines Revisited: A Design Space and Considerations for Expressive Storytelling. IEEE Transactions on Visualization and Computer Graphics 23, 9 (Sept 2017), 2151-2164. https://doi.org/10.1109/TVCG.2016.2614803

[11] M. Brehmer, B. Lee, P. Isenberg, and E. K. Choe. 2019. Visualizing Ranges over Time on Mobile Phones: A Task-Based Crowdsourced Evaluation. IEEE Transactions on Visualization and Computer Graphics 25, 1 (Jan 2019), 619-629. https://doi.org/10.1109/TVCG.2018.2865234

[12] M. Brehmer, B. Lee, P. Isenberg, and E. K. Choe. 2020. A Comparative Evaluation of Animation and Small Multiples for Trend Visualization on Mobile Phones. IEEE Transactions on Visualization and Computer Graphics 26, 1 (2020), 364-374. https://doi.org/10.1109/TVCG.2019.2934397

[13] Matthew Brehmer and Tamara Munzner. 2013. A Multi-Level Typology of Abstract Visualization Tasks. IEEE Transactions on Visualization and Computer Graphics 19, 12 (Dec. 2013), 2376-2385. https://doi.org/10.1109/TVCG.2013.124

[14] Stefano Burigat and Luca Chittaro. 2013. On the Effectiveness of Overview+Detail Visualization on Mobile Devices. Personal Ubiquitous Comput. 17, 2 (Feb. 2013), 371-385. https://doi.org/10.1007/s00779-011-0500-3

[15] T. Capin, K. Pulli, and T. Akenine-Möller. 2008. The State of the Art in Mobile Graphics Research. IEEE Computer Graphics and Applications 28, 4 (July 2008), 74-84. https://doi.org/10.1109/MCG.2008.83

[16] L. Chittaro. 2006. Visualizing information on mobile devices. Computer 39, 3 (March 2006), 40-45. https://doi.org/10.1109/MC.2006.109

[17] A. Cockburn, D. Ahlström, and C. Gutwin. 2012. Understanding performance in touch selections: Tap, drag and radial pointing drag with finger, stylus and mouse. International fournal of Human-Computer Studies 70, 3 (2012), 218-233. https://doi.org/10.1016/j.ijhcs.2011.11.002

[18] Sara Di Bartolomeo, Aditeya Pandey, Aristotelis Leventidis, David Saffo, Uzma Haque Syeda, Elin Carstensdottir, Magy Seif El-Nasr, Michelle A. Borkin, and Cody Dunne. 2020. Evaluating the Effect of Timeline Shape on Visualization Task Performance. In Proceedings of the 2020 CHI Conference on Human Factors in Computing Systems (Honolulu, HI, USA) (CHI '20). Association for Computing Machinery, New York, NY, USA, 1-12. https://doi.org/10.1145/3313831.3376237

[19] Leah Findlater, Joan Zhang, Jon E. Froehlich, and Karyn Moffatt. 2017. Differences in Crowdsourced vs. Lab-based Mobile and Desktop Input Performance Data. In Proceedings of the 2017 CHI Conference on Human Factors in Computing Systems (Denver, Colorado, USA) (CHI '17). ACM, New York, NY, USA, 6813-6824. https://doi.org/10.1145/3025453.3025820

[20] Paul M. Fitts. 1954. The information capacity of the human motor system in controlling the amplitude of movement. fournal of experimental psychology 47, 6 (June 1954), 381-391. https://doi.org/10.1037/0096-3445.121.3.262

[21] Clifton Forlines, Daniel Wigdor, Chia Shen, and Ravin Balakrishnan. 2007. Direct-touch vs. Mouse Input for Tabletop Displays. In Proceedings of the SIGCHI Conference on Human Factors in Computing Systems (San Jose, California, USA) (CHI '07). ACM, New York, NY, USA, 647-656. https://doi.org/10.1145/1240624.1240726

[22] Miguel A. García-Pérez. 1998. Forced-choice staircases with fixed step sizes: asymptotic and small-sample properties. Vision Research 38, 12 (1998), 1861 - 1881. https://doi.org/10.1016/S0042-6989(97)00340-4 
[23] Christian Holz and Patrick Baudisch. 2011. Understanding Touch. In Proceedings of the SIGCHI Conference on Human Factors in Computing Systems (Vancouver, BC, Canada) (CHI '11). ACM, New York, NY, USA, 2501-2510. https://doi.org/10.1145/1978942.1979308

[24] Tom Horak, Sriram Karthik Badam, Niklas Elmqvist, and Raimund Dachselt. 2018. When David Meets Goliath: Combining Smartwatches with a Large Vertical Display for Visual Data Exploration. In Proceedings of the 2018 CHI Conference on Human Factors in Computing Systems (Montreal QC, Canada) (CHI '18). Association for Computing Machinery, New York, NY, USA, 1-13. https://doi.org/10.1145/3173574.3173593

[25] ScientiaMobile Inc. 2017. Smartphone vs Tablet Orientation: Who's Using What? https://www.scientiamobile.com/page/smartphone-vs-tabletorientation-whos-using-what. Accessed: March 30, 2018.

[26] Alex M. Kale, Francis Nguyen, Matthew Kay, and Jessica Hullman. 2018. Hypothetical Outcome Plots Help Untrained Observers Judge Trends in Ambiguous Data. IEEE Transactions on Visualization and Computer Graphics 25 (2018), 892-902.

[27] Michael Krone, Christoph Müller, and Thomas Ertl. 2015. Remote Rendering and User Interaction on Mobile Devices for Scientific Visualization. In Proceedings of the 8th International Symposium on Visual Information Communication and Interaction (Tokyo, AA, Japan) (VINCI '15). Association for Computing Machinery, New York, NY, USA, 21-26. https://doi.org/10.1145/2801040.2801057

[28] R. Langner, T. Horak, and R. Dachselt. 2018. VisTiles: Coordinating and Combining Co-located Mobile Devices for Visual Data Exploration. IEEE Transactions on Visualization and Computer Graphics 24, 01 (jan 2018), 626-636. https://doi.org/10.1109/TVCG.2017.2744019

[29] Robert Mack and Kathy Lang. 1989. A Benchmark Comparison of Mouse and Touch Interface Techniques for an Intelligent Workstation Windowing Environment. Proceedings of the Human Factors Society Annual Meeting 33, 5 (1989), 325-329. https://doi.org/10.1177/154193128903300520 arXiv:https://doi.org/10.1177/154193128903300520

[30] William Playfair. 1786. The Commercial and Political Atlas and Statistical Breviary. Cambridge University Press, Cambridge, England.

[31] R. L. Potter, L. J. Weldon, and B. Shneiderman. 1988. Improving the Accuracy of Touch Screens: An Experimental Evaluation of Three Strategies. In Proceedings of the SIGCHI Conference on Human Factors in Computing Systems (Washington, D.C., USA) (CHI '88). Association for Computing Machinery, New York, NY, USA, 27-32. https://doi.org/10.1145/57167.57171

[32] Daniel Rosenberg and Anthony Grafton. 2010. Cartographies of Time: A History of the Timeline. Princeton Architectural Press, New York.

[33] Irene Ross. 2020. MobileVis. Retrieved Jan 9, 2021 from http://mobilev.is/

[34] Anne Roudaut, Stéphane Huot, and Eric Lecolinet. 2008. TapTap and MagStick: Improving One-Handed Target Acquisition on Small Touch-Screens. In Proceedings of the Working Conference on Advanced Visual Interfaces (Napoli, Italy) (AVI '08). Association for Computing Machinery, New York, NY, USA, 146-153. https://doi.org/10.1145/1385569.1385594

[35] Michail Schwab, Sicheng Hao, Olga Vitek, James Tompkin, Jeff Huang, and Michelle A. Borkin. 2019. Evaluating Pan and Zoom Timelines and Sliders. In Proceedings of the 2019 CHI Conference on Human Factors in Computing Systems (Glasgow, Scotland Uk) (CHI '19). ACM, New York, NY, USA, Article 556, 12 pages. https://doi.org/10.1145/3290605.3300786

[36] Katie A. Siek, Yvonne Rogers, and Kay H. Connelly. 2005. Fat Finger Worries: How Older and Younger Users Physically Interact with PDAs. In Proceedings of the 2005 IFIP TC13 International Conference on Human-Computer Interaction (Rome, Italy) (INTERACT'05). Springer-Verlag, Berlin, Heidelberg, 267-280. https://doi.org/10.1007/11555261_24

[37] Hendrik Sollich, Ulrich von Zadow, Tobias Pietzsch, Pavel Tomancak, and Raimund Dachselt. 2016. Exploring Time-Dependent Scientific Data Using Spatially Aware Mobiles and Large Displays. In Proceedings of the 2016 ACM International Conference on Interactive Surfaces and Spaces (Niagara Falls, Ontario, Canada) (ISS '16). Association for Computing Machinery, New York, NY, USA, 349-354. https://doi.org/10.1145/2992154.2996779

[38] M. Waldner, A. Diehl, D. Gračanin, R. Splechtna, C. Delrieux, and K. Matković. 2020. A Comparison of Radial and Linear Charts for Visualizing Daily Patterns. IEEE Transactions on Visualization and Computer Graphics 26, 1 (2020), 1033-1042. https://doi.org/10.1109/TVCG.2019.2934784

[39] Feng Wang and Xiangshi Ren. 2009. Empirical Evaluation for Finger Input Properties in Multi-touch Interaction. In Proceedings of the SIGCHI Conference on Human Factors in Computing Systems (Boston, MA, USA) (CHI '09). ACM, New York, NY, USA, 1063-1072. https://doi.org/10.1145/1518701.1518864

[40] Matt Whitlock, Keke Wu, and Danielle Albers Szafir. 2019. Designing for Mobile and Immersive Visual Analytics in the Field. IEEE Transactions on Visualization and Computer Graphics 26 (2019), 503-513. https://doi.org/10.1109/TVCG.2019.2934282

[41] Daniel Wigdor, Clifton Forlines, Patrick Baudisch, John Barnwell, and Chia Shen. 2007. Lucid Touch: A See-through Mobile Device. In Proceedings of the 20th Annual ACM Symposium on User Interface Software and Technology (Newport, Rhode Island, USA) (UIST'07). ACM, New York, NY, USA 269-278. https://doi.org/10.1145/1294211.1294259 